\title{
The Motor-cognitive Training on Cognition and Physical Performance in the Older Adults with Mild Cognition Impairment : A Literature Review
}

\author{
Jihye Jung ${ }^{a}{ }^{\oplus}$, Yong-Seong Kim ${ }^{b}(D)$ Seungwon Lee ${ }^{a, c^{*}}(\mathbb{b})$ \\ a Institute of SMART Rehabilitation, Sahmyook University

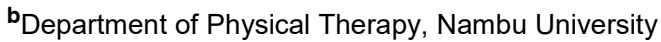 \\ 'Department of Physcial Therapy, College of Health and Welfare, Sahmyook University
}

Objective: Older adults with mild cognitive impairment (MCI) are more likely to progress to dementia. Motor-cognitive training is applied as a dual-task to improve the cognitive and physical functions of older adults with MCI. The purpose of the study was to know the recent trends in motor training types and outcome measures used for motor-cognitive training in older adults with MCI

Design: Aliterature review

Methods: This literature review was conducted in Pubmed, MEDLINE® and Google Scholar with the following key words: older adults, mild cognitive impairment, motor-cognitive training, cognition, and dual-task. The 7 studies were found with the search tool and all studies were randomized controlled trials.

Results: In motor-cognitive training in older adults with MCI, 6 studies applied aerobic exercise. And 3 out of 6 studies also applied strengthening exercises. One study applied dual tasks without aerobic exercise. In the 6 studies, overall cognitive and executive function were used as outcome measures, and physical function was evaluated as gait performance. Memory and physical frailty were also used as measurement tools. As a result of all studies, when motor-cognitive training was applied, cognition and physical performance showed significant results.

Conclusions: A recent five-year study applied mainly aerobic exercise and strength training to older adults with MCI and found it to improve cognitive and physical performance.

Key Words: Cognition, Aged, Cognitive Dysfunction, Exercise Therapy

\section{Introduction}

Mild cognitive impairment $(\mathrm{MCI})$ is an important predictive risk factor for dementia [1,2] and is an intermediate stage between normal aging and dementia [3]. Although active daily living(ADL) function can be maintained [4] motor impairment such as cognitive decline, gait and balance deterioration is a typical characteristic of the older adults with MCI [5,6]. Older adults with MCI are more likely to progress to dementia [7] and the conversion rate from MCI is depending on diagnostic criteria and assessment tools, the incidence of dementia is estimated at $10 \%$ to $15 \%$ per year [8].

The older adults with MCI complain of difficulties in situations where they have to multitask in real life because they have a memory impairment [9]. Daily life living in real life is complex, and most are given more than dual-task situations. Previous studies mainly applied the single-task of cognitive training or motor training [10-13], but recently studies related to the dual task are being conducted.

Received: Dec 20, 2021 Revised: Dec 29, 2021 Accepted: Dec 29, 2021

Corresponding author: Seungwon Lee (ORCID https://orcid.org/0000-0002-0413-0510)

Department of Physical Therapy, College of Health and Welfare, Sahmyook University

815 Hwarang-ro, Nowon-gu, Seoul 01795, Republic of Korea

Tel: +82-2-3399-1630 Fax:+82-2-3399-1639 E-mail: swlee@syu.ac.kr

This is an Open-Access article distributed under the terms of the Creative Commons Attribution Non-Commercial License (http://creativecommons.org/licenses/ by-nc/4.0) which permits unrestricted non-commercial use, distribution, and reproduction in any medium, provided the original work is properly cited.

Copyright $(2021$ Korean Academy of Physical Therapy Rehabilitation Science 
There are previous studies that showed the effect of cognitive ability and physical function by applying exercise training with cognitive training for cognitive improvement of the older adults [9,14,15]. Motor-cognitive training is beneficial to enhance cognitive and physical function even for older adults with MCI $[3,16]$. In the method of applying motor-cognitive training, applying tasks at the simultaneous has an advantage in cognitive improvement than applying them sequentially [17].

Recently, dual-task training using virtual reality (VR) [18,19] and aerobic exercise [20] have been mainly implemented for motor-cognitive training. Aerobic exercise significantly increases functional activation of MCI brain regions and helps to effectively improve cognitive function [21]. The improvement of physical function and cognitive ability through exercise training has raised the possibility that biological pathways and neural substrates may be shared between them, but the exact mechanism has not yet been elucidated [9,22]. Nevertheless, studies that improve cognition through exercise training continue to be published.

Therefore, this study intends to identify the latest trends by reviewing recent studies related to motor-cognitive training, which is mainly conducted in an environment similar to real life. And to suggest what the ideal motor-cognitive training is for in the older adults with MCI.

\section{Methods}

\section{Data sources and study criteria}

The collection of theses was conducted by 3 physical therapists using selected keywords, and the collected documents were organized, compared, and analyzed through a bibliographic information program (EndNote 20, Thomson Reuters, USA). In the case of inconsistency between the abstract and the main text of the thesis during the selection process, it was excluded from the collection process(Figure 1).

The study criteria used in the analysis of this study

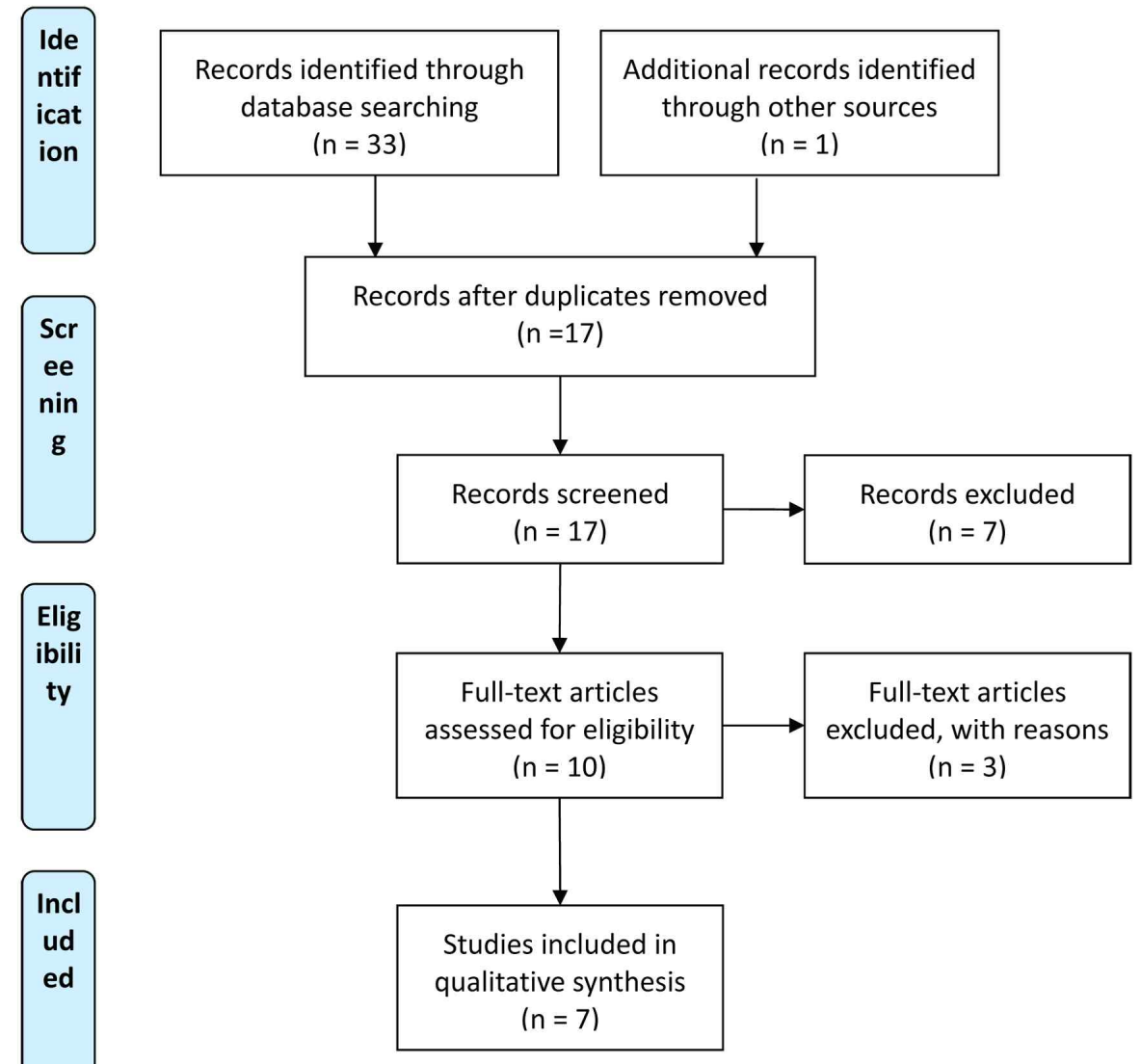

Figure 1. flow diagram 
were as follows: (1) studies in which subjects were diagnosed with MCI, (2) a study applying dual-task for motorcognitive training, (3) a study that included cognitive and physical function as an outcome according to each intervention method, (4) a study conducted as a randomized control trails study. In addition, studies on cognitive enhancement of neurological diseases other than MCI, a study written in languages other than English and Korean, and studies that could not accurately explain the effect of motor-cognitive training due to the study design were excluded. In this literature review study, studies from 2016 were searched using the databases of PubMed, MEDLINE® and Google Scholar.

\section{Search terms}

The keywords used in the search were as follows and were used either in combination or independently: 'older adults', 'elderly', 'elderly cognition', 'cognitive decline', 'mild cognitive impairment', 'motor-cognitive training', 'exercise', 'aerobic exercise', 'physical training', 'dual-task', 'cognition', 'cognitive task', and 'physical function'. As a result, the 7 studies were found with the search tool and all studies were randomized controlled trials.

\section{Study selection process}

For studies collected through the database, duplicate studies were removed using a reference management program (EndNote 20, Thomson Reuters, USA). After screening through the abstract and title, the original text of the thesis was reviewed according to the selection criteria. In the process of data extraction, general characteristics, specific interventions, and research results were extracted. If the data do not match, the original text was reviewed and finally selected and summarized.

\section{Results}

According to our selection criteria, 7 studies were classifiedusing the PICO search strategy(Table 1) (Figure 1). As a result of all studies, when motor -cognitive training was applied, cognition and physical performance showed significant results.

In motor-cognitive training in older adults with
MCI, 6 studies applied aerobic exercise. And 3 out of 6 studies also applied strengthening exercises. One study applied dual tasks without aerobic exercise.

In study of Park JH [23], motor training in motor -cognitive training was applied simultaneously with cognitive training such as range of motion (ROM), TheraBand strengthening (low intensity), passing/throwing a ball. And for aerobic exercise, wrist, elbow, shoulder, ankle, knee range of motion exercise and walking motion were sequentially applied for during intervention program.

Kwan et al. [19] was the under-desk ergometer with adjustable cycling resistance was applied alone without combining with other exercises as a motor training during cognitive training using virtual reality program.

Park HT et al. [3] was motor training, aerobic exercise combined activity program was applied. The combined activity program includes a warm-up, stretching training, aerobic exercise, balance exercise, aerobic and cognitive training, resting time with feedback and encouraging active daily living physical education, and cool-down exercise. And the aerobic exercise applied was stair stepping, endurance walking and stair climbing, and walking on an agility ladder. During aerobic exercise, the target heart rate zone was at $55 \%$ to $80 \%$ of the maximum heart rate in the intervention [24].

Lia et al. [18] were also applied motor training combined with aerobic exercise. And the program was composed of a simplified 24-form Yang-style Tai Chi, resistance exercise, aerobic exercise, ADL relating functional tasks, and included for that to improve upper and lower extremity balance, stability, strength, and endurance.

The motor training applied in the study of Law et al. [25] is warm-up of light stretching, moderate-intensity aerobic exercise, whole-body movement exercise, bicycle, arm ergometry, cool-down exercise. The functional task exercise group was appliedwarm-up, core functional tasks exercise, and cool-down exercise.

Shimada et al. [8] was applied an activity program that combines aerobic exercise, and also included muscle strengthening, and postural balance training. Aerobic exercise includes climbing stairs, endurance walking, and walking on a balance board. Average aerobic exercise intensity was $60 \%$ to $80 \%$ of maximum heart rate (HR) identically with previous studies [24]. 


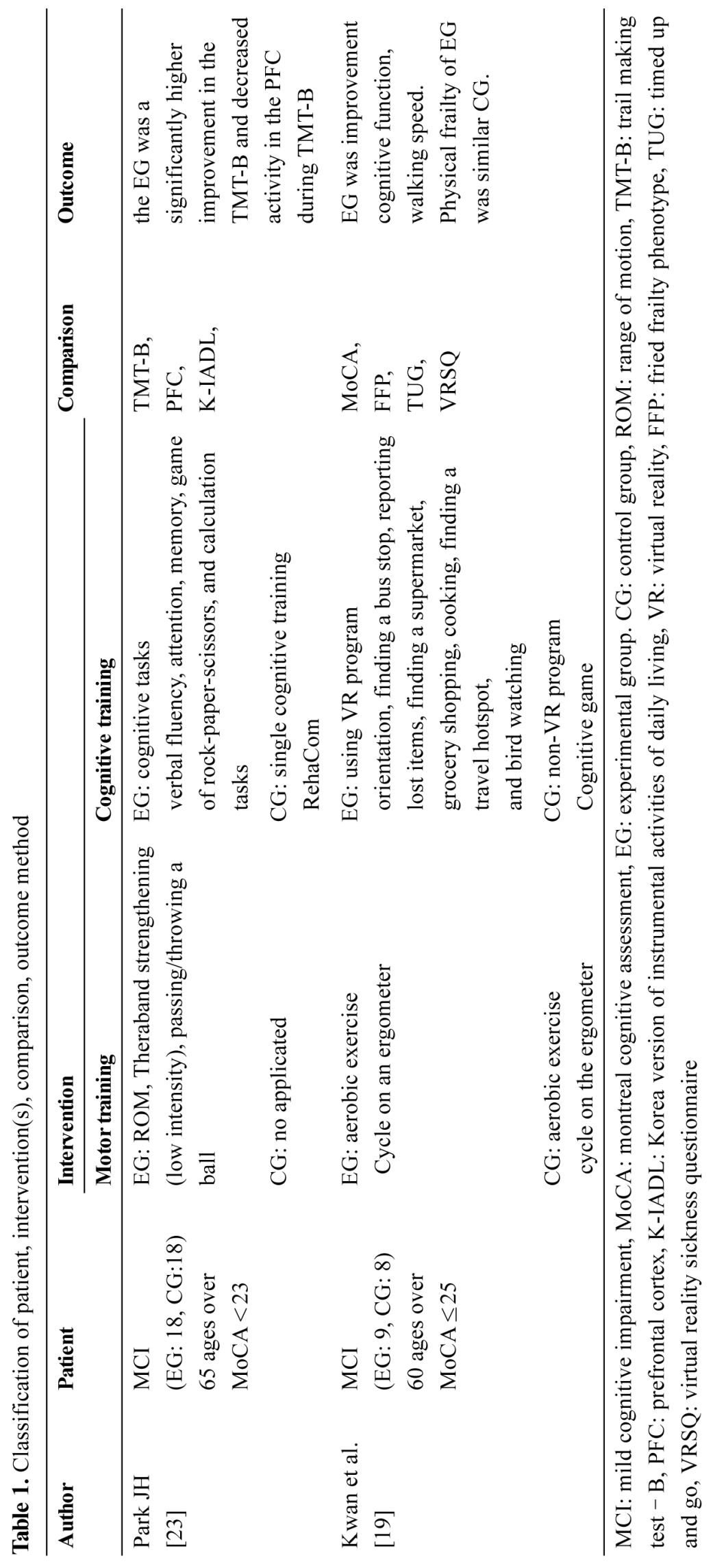




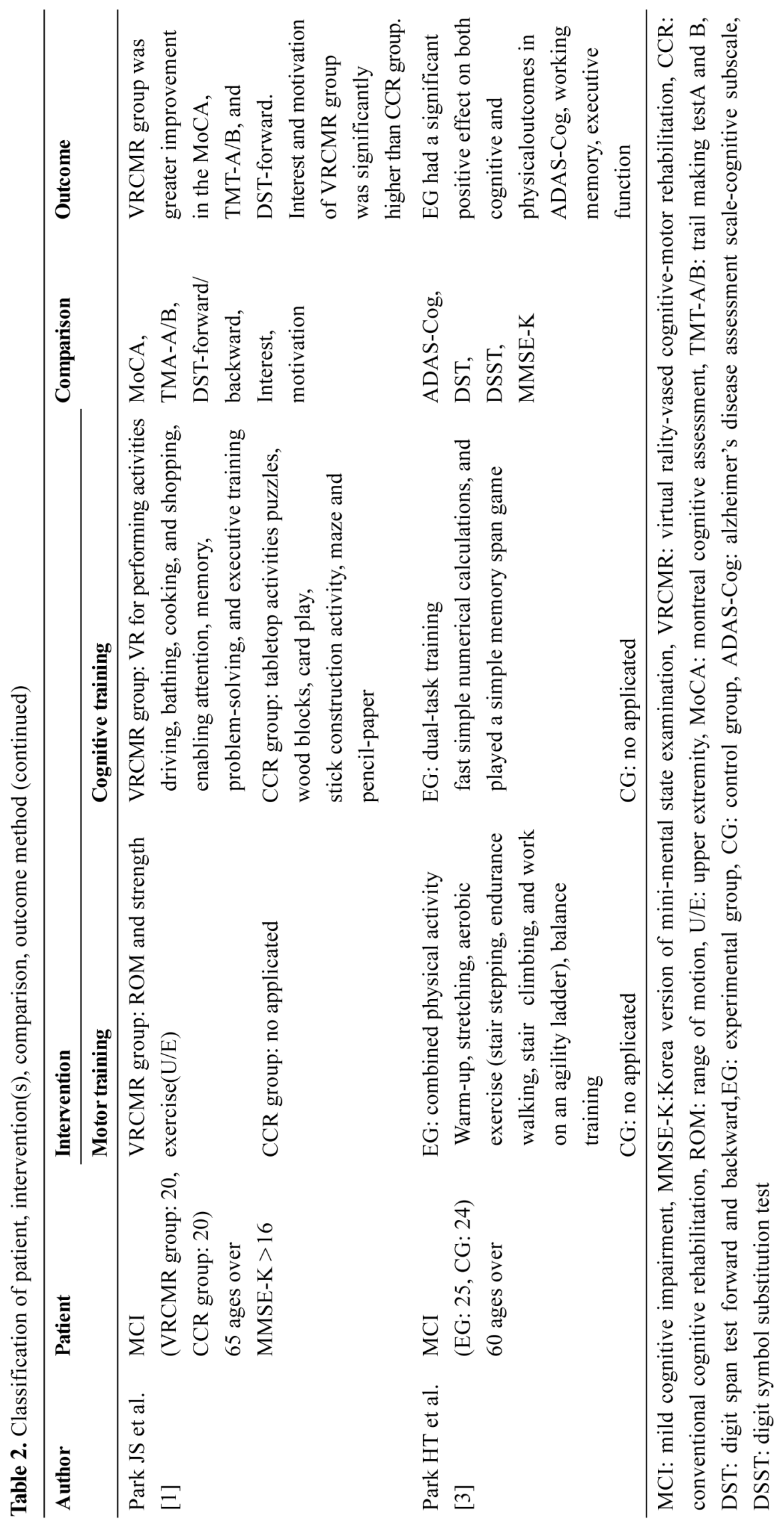




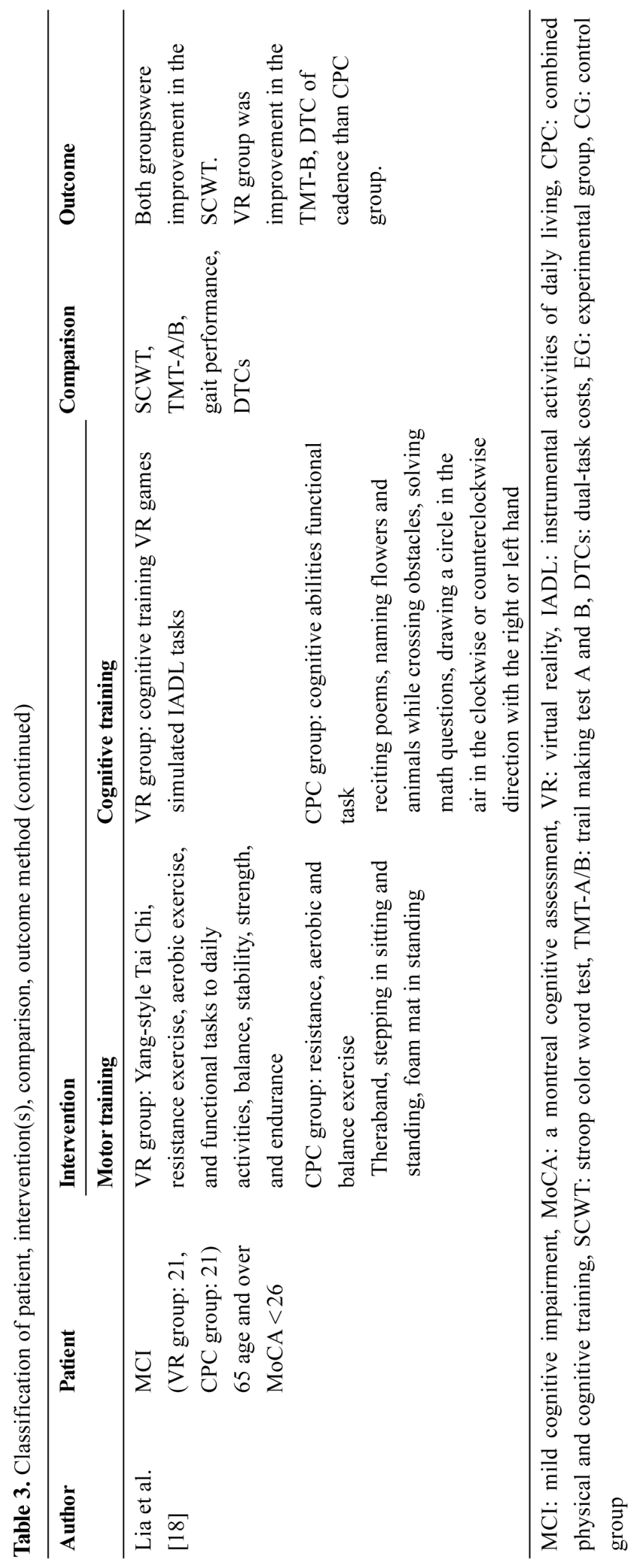




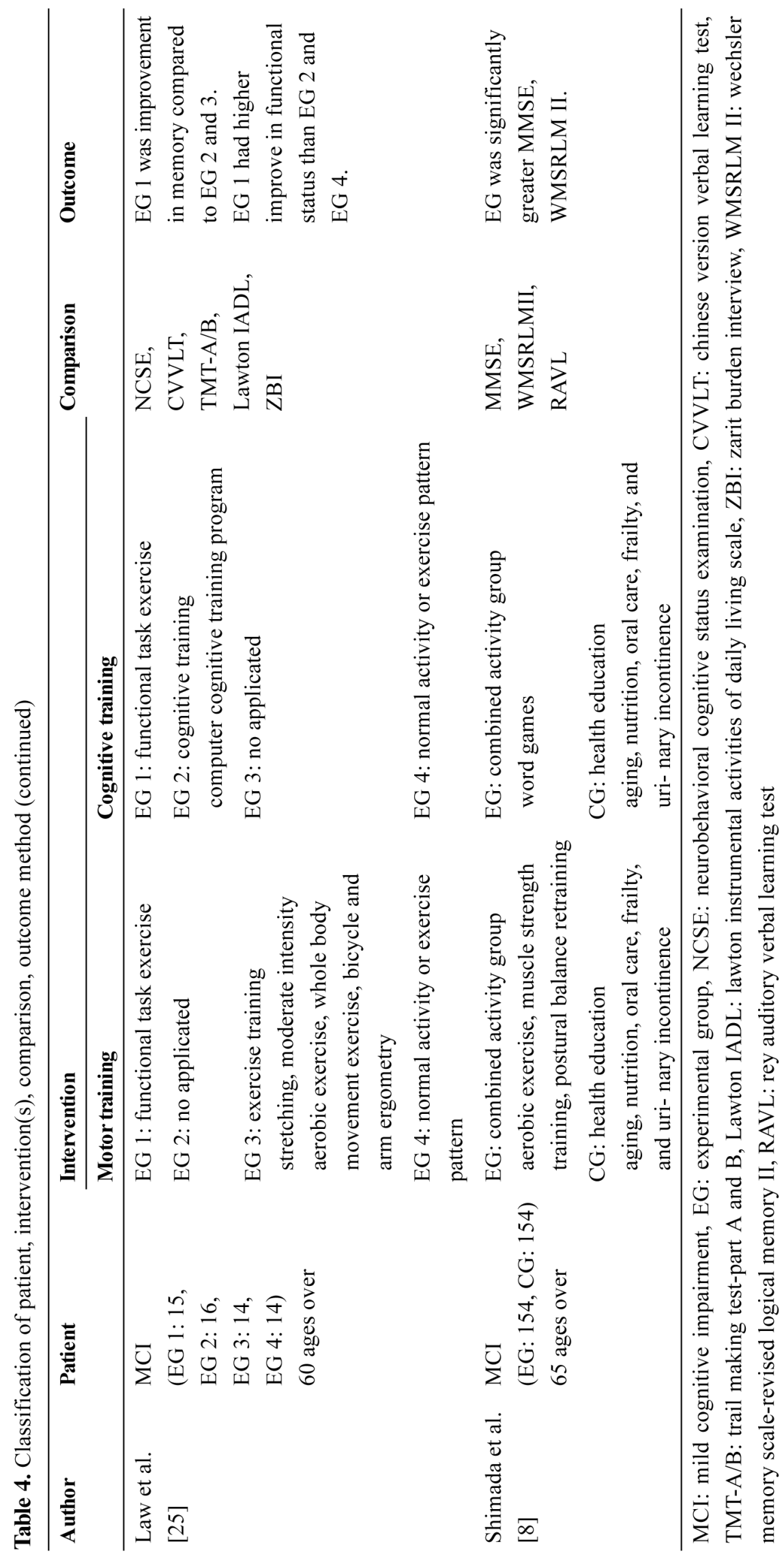


In the 6 studies, overall cognitive and executive function were used as outcome measures, and physical function was evaluated as gait performance. Memory and physical frailty were also used as measurement tools. Of the 7 studies, 3 studies [18,19,23] were using a montreal cognitive assessment (MoCA) as eligibility criteria were, and 1 study [1] was using a Korean version of mini-mental state examination (MMSE-K). For cognitive evaluation of motor-cognitive training, a trail making test A and B (TMT-A/B), the stroop color-word test (SCWT) to evaluate executive function were mainly used, and MoCA, modified alzheimer's disease assessment scale-cognitive subscale (ADAS-Cog), and MMSE were used for overall popular intelligence. Memory function was evaluated by a digit span test forward and backward (DST) and Chinese version verbal learning test (CVVLT).

\section{Discussion}

As a result of analyzing a recent study applied for motor-cognitive training to older adults with MCI, it was confirmed that the dual-task was applied simultaneously, and that motor training was applied either alone or in combination with aerobic exercise as an activity program. Therefore, simultaneous application of aerobic exercise and cognitive tasks will be an intervention method that can lead to improvement in cognitive ability and physical function.

In the study of Park HT et al. [3], the physical and cognitive combined intervention program applied increased the prefrontal cognitive function of MCI, and Shimada et al. [8] was find out the volume loss in the left brain hemisphere was smaller than the right during of the motor-cognitive exercise. Park JH [23] described a significant decrease in $\mathrm{HbO}_{2}$ in the prefrontal lobe during executive function tests as an increase in the neural efficiency. According to the results of these previous studies, the brain volume over 65 years of age decreases by $0.5 \%$ to $1 \%$ annually due to normal aging, and the hippocampal volume decreases by $1 \%$ to $2 \%$ even in the absence of dementia [26], resulting in a higher risk of cognitive impairment. Therefore motor-cognitive training could expect applied as a good arbitration method.In particular, executive function can improve through aerobic exercise, and neural activation improves neural efficiency in areas where aerobic training supports executive processes of selective attention and response inhibition [27].

The MoCA, which was used the most in previous studies analyzed in this study, was developed to distinguish mild cognitive impairment [28] and is said to show higher diagnostic accuracy than MMSE [29,30]. And a TMT-A/B used to evaluate executive function is the most famous neuropsychological test and can be applied in various ways considering age and educational level [31]. It was confirmed that most studies evaluated cognitive function using similar outcome measures.

A recent motor-cognitive training study for MCI was conducted in virtual reality, but it was also conducted with content related to daily life tasks. In any environment, positive effects of cognitive ability and physical ability can be derived by applying the combination of body movements including aerobic exercise and tasks related to real life. It is believed that good results can be drawn if future experimental studies are conducted with these results as a reference.

\section{Acknowledgement}

This research was supported by Basic Science Research Program through the National Research Foundation of Korea (NRF) funded by the Ministry of Education (NRF-2021R1F1A1060798).

\section{Conflict of interest}

The authors of this study declare that there are no potential conflicts of interest with respect to the research, authorship, and publication.

\section{References}

1. Park J-S, Jung Y-J, Lee G. Virtual Reality-Based Cognitive-Motor Rehabilitation in Older Adults with Mild Cognitive Impairment: A Randomized Controlled Study on Motivation and Cognitive Function. Healthcare. 2020;8:335.

2. Livingston G, Sommerlad A, Orgeta V, Costafreda SG, Huntley J, Ames D, et al. Dementia prevention, intervention, and care. Lancet. 2017;390: 2673-734. 
3. Park HT, Park JH, Na HR, Hiroyuki S, Kim GM, Jung MK, et al. Combined Intervention of Physical Activity, Aerobic Exercise, and Cognitive Exercise Intervention to Prevent Cognitive Decline for Patients with Mild Cognitive Impairment: A Randomized Controlled Clinical Study. J Clin Med. 2019;8:940.

4. Tarumi T, Rossetti H, Thomas BP, Harris T, Tseng BY, Turner M, et al. Exercise Training in Amnestic Mild Cognitive Impairment: A One-Year Randomized Controlled Trial. J Alzheimer's Dis. 2019;71:421-33.

5. Tangen GG, Engedal K, Bergland A, Moger TA, Mengshoel AM. Relationships Between Balance and Cognition in Patients With Subjective Cognitive Impairment, Mild Cognitive Impairment, and Alzheimer Disease. Phys Ther. 2014;94:1123-34.

6. Doi T, Makizako H, Shimada H, Yoshida D, Tsutsumimoto K, Sawa R, et al. Effects of multicomponent exercise on spatial-temporal gait parameters among the elderly with amnestic mild cognitive impairment (aMCI): preliminary results from a randomized controlled trial (RCT). Arch Gerontol Geriatr. 2013;56:104-8.

7. Petersen RC. Mild cognitive impairment as a diagnostic entity. J Intern Med. 2004;256:183-94.

8. Shimada H, Makizako H, Doi T, Park H, Tsutsumimoto $\mathrm{K}$, Verghese J, et al. Effects of Combined Physical and Cognitive Exercises on Cognition and Mobility in Patients With Mild Cognitive Impairment: A Randomized Clinical Trial. J Am Med Dir Assoc. 2018;19:584-91.

9. Falck RS, Davis JC, Best JR, Crockett RA, Liu-Ambrose T. Impact of exercise training on physical and cognitive function among older adults: a systematic review and meta-analysis. Neurobiol Aging. 2019;79:119-30.

10. McDougall GJ, McDonough IM, LaRocca M. Memory training for adults with probable mild cognitive impairment: a pilot study. Aging Ment Health. 2019;23:1433-41.

11. Giuli C, Papa R, Lattanzio F, Postacchini D. The Effects of Cognitive Training for Elderly: Results from My Mind Project. Rejuvenation Res. 2016; 19:485-94.

12. Mudar RA, Chapman SB, Rackley A, Eroh J, Chiang HS, Perez A, et al. Enhancing latent cognitive capacity in mild cognitive impairment with gist rea- soning training: a pilot study. Int J Geriatr Psychiatry. 2017;32:548-55

13. Zhu Y, Wu H, Qi M, Wang S, Zhang Q, Zhou L, et al. Effects of a specially designed aerobic dance routine on mild cognitive impairment. Clin Interv Aging. 2018;13:1691-700.

14. Murata S, Ono R, Yasuda H, Tanemura R, Kido Y, Kowa H. Effect of a Combined Exercise and Cognitive Activity Intervention on Cognitive Function in Community-dwelling Older Adults: A Pilot Randomized Controlled Trial. Phys Ther Res. 2021;24:112-9.

15. ten Brinke LF, Best JR, Chan JLC, Ghag C, Erickson KI, Handy TC, et al. The Effects of Computerized Cognitive Training With and Without Physical Exercise on Cognitive Function in Older Adults: An 8-Week Randomized Controlled Trial. The Journals of Gerontology: Series A. 2019;75:755-63.

16. Law C-K, Lam FMH, Chung RCK, Pang MYC. Physical exercise attenuates cognitive decline and reduces behavioural problems in people with mild cognitive impairment and dementia: a systematic review. J Physiother. 2020;66:9-18.

17. Tait JL, Duckham RL, Milte CM, Main LC, Daly RM. Influence of Sequential vs. Simultaneous Dual-Task Exercise Training on Cognitive Function in Older Adults. Front Aging Neurosci. 2017;9.

18. Liao Y-Y, Chen I-H, Lin Y-J, Chen Y, Hsu W-C. Effects of Virtual Reality-Based Physical and Cognitive Training on Executive Function and Dual-Task Gait Performance in Older Adults With Mild Cognitive Impairment: A Randomized Control Trial. Front Aging Neurosci. 2019;11.

19. Kwan RYC, Liu JYW, Fong KNK, Qin J, Leung PK-Y, Sin OSK, et al. Feasibility and Effects of Virtual Reality Motor-Cognitive Training in Community -Dwelling Older People With Cognitive Frailty: Pilot Randomized Controlled Trial. JMIR Serious Games. 2021;9:e28400.

20. Makino $\mathrm{T}$, Umegaki $\mathrm{H}$, Ando $\mathrm{M}$, Cheng XW, Ishida $\mathrm{K}$, Akima $\mathrm{H}$, et al. Effects of Aerobic, Resistance, or Combined Exercise Training Among Older Adults with Subjective Memory Complaints: A Randomized Controlled Trial. J Alzheimer's Dis. 2021;82:701-17.

21. Qi M, Zhu Y, Zhang L, Wu T, Wang J. The effect of aerobic dance intervention on brain spontaneous 
activity in older adults with mild cognitive impairment: A resting-state functional MRI study. Exp Ther Med. 2019;17:715-22.

22. Rea IM. Towards ageing well: Use it or lose it: Exercise, epigenetics and cognition. Biogerontology. 2017;18:679-91.

23. Park J-H. Effects of Cognitive-Physical Dual-Task Training on Executive Function and Activity in the Prefrontal Cortex of Older Adults with Mild Cognitive Impairment. Brain Neurorehabil. 2021;14.

24. Angevaren M, Aufdemkampe G, Verhaar HJ, Aleman A, Vanhees L. Physical activity and enhanced fitness to improve cognitive function in older people without known cognitive impairment. Cochrane Database Syst Rev. 2008:Cd005381.

25. Law LLF, Mok VCT, Yau MMK. Effects of functional tasks exercise on cognitive functions of older adults with mild cognitive impairment: a randomized controlled pilot trial. Alzheimer's Res Ther. 2019;11.

26. Jack CR, Jr., Wiste HJ, Vemuri P, Weigand SD, Senjem ML, Zeng G, et al. Brain beta-amyloid measures and magnetic resonance imaging atrophy both predict time-to-progression from mild cognitive impairment to Alzheimer's disease. Brain. 2010;133:3336-48.

27. Hsu CL, Best JR, Davis JC, Nagamatsu LS, Wang $\mathrm{S}$, Boyd LA, et al. Aerobic exercise promotes executive functions and impacts functional neural activity among older adults with vascular cognitive impairment. Br J Sports Med. 2018;52:184-91.

28. Nasreddine ZS, Phillips NA, Bédirian V, Charbonneau S, Whitehead V, Collin I, et al. The Montreal Cognitive Assessment, MoCA: a brief screening tool for mild cognitive impairment. J Am Geriatr Soc. 2005;53:695-9.

29. Freitas S, Simões MR, Alves L, Santana I. Montreal Cognitive Assessment: influence of sociodemographic and health variables. Arch Clin Neuropsychol. 2012;27:165-75.

30. Larner AJ. Screening utility of the Montreal Cognitive Assessment (MoCA): in place of -or as well as the MMSE? Int Psychogeriatr. 2012;24:391-6.

31. Tombaugh T. Trail Making Test A and B: Normative data stratified by age and education. Arch Clin Neuropsychol. 2004;19:203-14. 